\title{
Color Change of Sudan III against Concentrated Sulfuric Acid in Acetonitrile and Quantification for a Small Amount of Concentrated Sulfuric Acid
}

\author{
Takao SaKuraI, ${ }^{* * * \dagger}{ }^{*}$ Shoji Kurata,* and Kenji OgIno** \\ *Criminal Investigation Laboratory, Metropolitan Police Department, 2-1-1 Kasumigaseki, Chiyoda, \\ Tokyo 100-8929, Japan \\ **Graduate School of Bio-applications and Systems Engineering, Tokyo University of Agriculture and \\ Technology, 2-24 Nakamachi, Koganei, Tokyo 184-8558, Japan
}

\begin{abstract}
The color-changing phenomenon of hydrophobic bisazo dye, Sudan III in an acetonitrile solution against the addition of concentrated sulfuric acid has been discovered and the chromic properties investigated. Based on observations, a novel quantification method of concentrated sulfuric acid has been developed. Sudan III changes its color from orange to blue against a small volume of sulfuric acid, and the acetonitrile solution of Sudan III is the most suitable for observing the color-change phenomenon. ${ }^{1} \mathrm{H}-\mathrm{NMR}$ and UV-Vis spectroscopic studies showed that the color-change mechanism of Sudan III against sulfuric acid is due to the protonation of the dye by sulfuric acid. This phenomenon is applicable to the quantification of concentrated sulfuric acid by introducing the Hammett acidity function. The proposed method requires only a small amount of the sample, $0.04 \mathrm{~mL}$, and enables rapid quantification.
\end{abstract}

Keywords Sudan III, sulfuric acid, quantification method

(Received June 6, 2015; Accepted August 4, 2015; Published February 10, 2016)

\section{Introduction}

Concentrated sulfuric acid is widely used in chemical laboratories and industrial plants. In industry, it is used in a variety of fields, such as catalysts for the production of highoctane-number trimethylheptanes ${ }^{1}$ and biodiese ${ }^{2,3}$ and solvents for the production of fibers with high mechanical strength, ${ }^{4}$ for example. On the other hand, concentrated sulfuric acid is an extremely hazardous chemical, and it sometimes causes fatal injuries. ${ }^{5}$ In the criminal fields, it is abused in some assaults, clandestine methamphetamine manufacture ${ }^{6}$ or in the production of counterfeit gas oils in Japan. ${ }^{7,8}$ Although the titration method has been generally utilized for the quantification of concentrated sulfuric acid, it is not suited to high-throughput analyses because it is time-consuming and requires a special technique and apparatus. In criminal fields, there are difficulties in analyzing sulfuric acid: 1) rapidity is required; 2) the amount of available sample is usually small; 3) the sample concentration is unpredictable before analysis. Therefore, a rapid and simple analytical method for the quantification of small amounts of concentrated sulfuric acid is strongly desired in forensic analyses, and it will also be helpful in industrial fields for quality checks. To date, however, an efficient method has not yet been developed.

Some sensing methods for concentrated acids using the color change of dyes have already been reported. For example,

† To whom correspondence should be addressed.

E-mail: 50012701192@st.tuat.ac.jp
Wang et al. investigated acid-sensing applications of 9-(cycloheptatrienylidene)fluorine in the range of $0-4 \mathrm{M} .^{9}$ Our previous paper reported on an optical sensing method using Oil Red O supported by florisil, which enables one to predict the approximate concentration of sulfuric acid in the range of 0.5 $12 \mathrm{M}$ by visual observation. ${ }^{10}$ In our previous method, however, it is difficult to discriminate concentrations over $12 \mathrm{M}$; also, the mechanism for the color change of Oil Red $\mathrm{O}$ has not been understood.

A hydrophobic bisazo dye, Sudan III (C.I. solvent red 23) has a simpler structure than does Oil Red O (Scheme 1). We found that these dyes show similar color-change properties against concentrated sulfuric acid. Because of the presence of four methyl groups, Oil Red O has isomeric structures, which leads to difficulties in the elucidation of its color-change mechanism. Due to its structural simplicity, we choose Sudan III to investigate the color-change mechanism. Sudan III is known as a kind of Sudan dye, which is mainly used for staining lipids or the coloring of waxes, oils, cosmetics and foods. ${ }^{11-14}$ In this

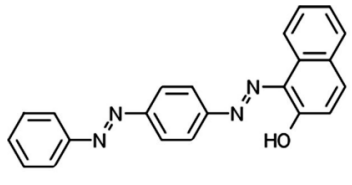

Sudan III

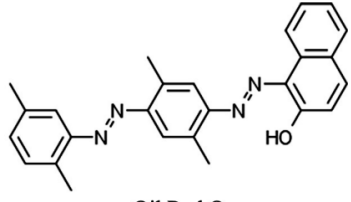

Oil Red O
Scheme 1 Structures of Sudan III and Oil Red O. 
report, the color-change behavior of Sudan III against sulfuric acid in acetonitrile was investigated. UV-Vis and ${ }^{1} \mathrm{H}-\mathrm{NMR}$ spectrometries provided information about the color-change mechanism. Using this color-change phenomenon, we propose a new method for the quantification of a small amount of concentrated sulfuric acid. The method requires only a small amount of the sample, $0.04 \mathrm{~mL}$, and enables rapid quantification.

\section{Experimental}

\section{Reagents and chemicals}

Sudan III, organic solvents (acetonitrile methanol, ethanol, 2-propanol, acetone, dimethyl sulfoxide), acids (sulfuric acid, hydrochloric acid, hydrofluoric acid, nitric acid, phosphoric acid, formic acid and acetic acid) were purchased from Wako (Japan). Sodium hydroxide was purchased from Kanto Kagaku (Japan). For ${ }^{1} \mathrm{H}-\mathrm{NMR}$ measurements, deuterated acetonitrile and tetramethylsilane purchased from Wako (Japan) were used as a solvent and an internal reference, respectively. All reagents were used as received. For quantification, the concentration of sulfuric acid was determined to be $17.99 \mathrm{M}$ by titration using a $0.54 \mathrm{M}$ of $\mathrm{NaOH}$ aqueous solution.

\section{Apparatus}

${ }^{1} \mathrm{H}-\mathrm{NMR}$ spectra were measured with a JEOL AL300 spectrometer (JEOL Ltd., Japan). UV-Vis spectra were measured with a JASCO V-670 spectrophotometer (JASCO Corp., Japan) using a quartz cell with a path length of $10 \mathrm{~mm}$.

\section{Procedure}

For ${ }^{1} \mathrm{H}-\mathrm{NMR}$ measurements, $0.6 \mathrm{mg}$ of Sudan III was brought to deuterated acetonitrile containing $0.01 \mathrm{vol} \%$ of tetramethylsilane (TMS). Solutions with or without sulfuric acid were transferred to NMR tubes with a 5-mm inner diameter.

The proton spectra were recorded at $303.0 \mathrm{~K}$ with accumulation time of 32. The TMS signal was used as a reference signal. Data were processed and analyzed with Delta software (JEOL Ltd., Japan).

For UV-Vis measurements, solutions of Sudan III were prepared as follows. A stock solution was prepared by dissolving $2.5 \mathrm{mg}$ of Sudan III in $50.0 \mathrm{~mL}$ of acetone. Then, $0.40 \mathrm{~mL}$ of the stock solution was heated in glass vials to evaporate acetone. Acetone was chosen as the solvent of the stock solution because of the good solubility of Sudan III and easiness of evaporation. To investigate the color-change property of Sudan III, each organic solvent was added to the vial so as to dissolve the dye powder; some amount of acids were then added. The final volume of each solution was adjusted to $5.0 \mathrm{~mL}$. To quantify the sulfuric acid, $5.0 \mathrm{~mL}$ of acetonitrile was added to the vial to yield the acetonitrile solution of Sudan III. Then, $0.04 \mathrm{~mL}$ of sulfuric acid with various concentrations was added to the dye solution, followed by the measurement. UV-Vis absorption spectra were recorded using acetonitrile without any dye as a background. All measurements were carried out at room temperature (approximately $298 \mathrm{~K}$ ).

\section{Results and Discussion}

\section{NMR spectroscopic study}

To elucidate the color-change mechanism of Sudan III against sulfuric acid, ${ }^{1} \mathrm{H}-\mathrm{NMR}$ measurements were carried out. By visual observation, the orange color of a deuterated acetonitrile solution of Sudan III turned to dark blue when approximately
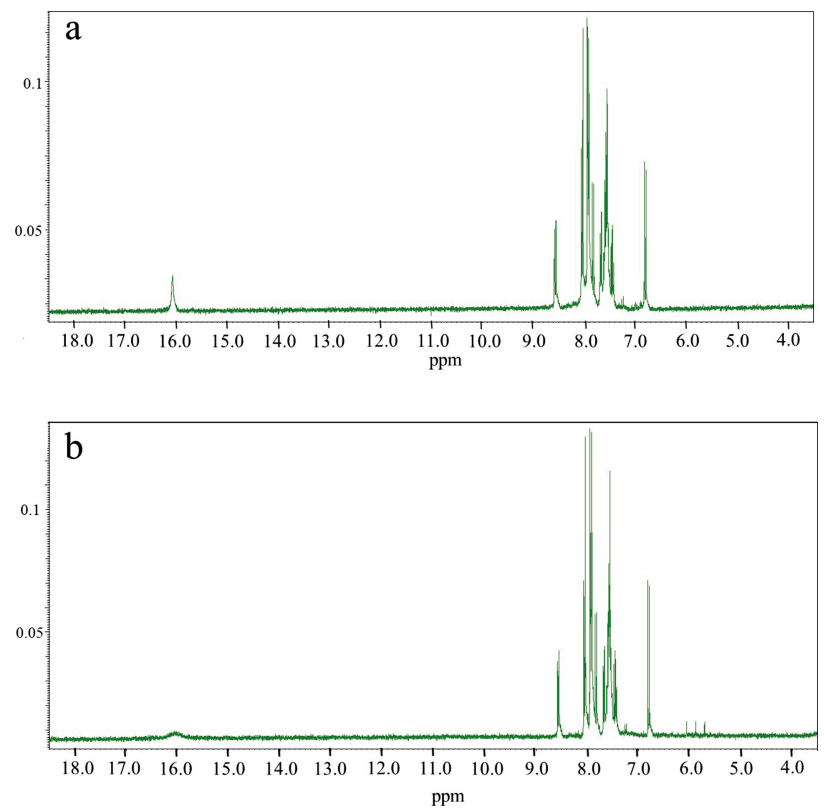

Fig. $1 \quad{ }^{1} \mathrm{H}$-NMR spectra of deuterated acetonitrile solution of Sudan III without (a) or with the presence (b) of sulfuric acid.

$2 \mu \mathrm{L}$ of sulfuric acid was added. The ${ }^{1} \mathrm{H}-\mathrm{NMR}$ spectra of a deuterated acetonitrile solution of Sudan III without or with sulfuric acid are shown in Figs. 1a and 1b, respectively. Referring to the literature, ${ }^{14-16}$ the observed peaks were assigned as follows. Resonances at between $6.5-9 \mathrm{ppm}$ are due to aromatic protons. A relatively broad resonance at $16.08 \mathrm{ppm}$ in Fig. 1a is due to the azo-hydrazone tautomeric proton. Resonances due to aromatic protons did not change after the addition of sulfuric acid. On the other hand, a resonance due to the tautomeric proton was immediately broadened after the addition of sulfuric acid (sulfuric acid concentration of the solution, 0.04 vol\%), as shown in Fig. 1b. This indicates that a rapid hydrogen exchange occurred between sulfuric acid and the tautomeric proton of Sudan III. Although this result does not give any direct information about the color-change mechanism, it suggests that a proton exchange or protonation in the azo or hydroxyl group is the key role of the color-change phenomenon. When other acids, such as formic acid and acetic acid, were added in place of sulfuric acid, no change was observed for a resonance at $16.08 \mathrm{ppm}$. This suggests that there is almost no interaction between the acids and the tautomeric proton of the dye.

\section{Color change property of Sudan III}

A series of observations of the color-change property of Sudan III for various volumes of sulfuric acid were carried out. When $0-0.1 \mathrm{~mL}$ of sulfuric acid was added to an acetonitrile solution of Sudan III, the color of the solution changed clearly from orange to blue upon the addition of sulfuric acid. Figure 2 shows the UV-Vis spectra of acetonitrile solutions of Sudan III without and with various amounts of sulfuric acid. For an acetonitrile solution of Sudan III without sulfuric acid, absorption peaks at 505 and $350 \mathrm{~nm}$ were observed. Upon the addition of sulfuric acid, a peak at $611 \mathrm{~nm}$ was observed. Absorbances at 505 and $350 \mathrm{~nm}$ were decreased, while the absorption at $611 \mathrm{~nm}$ was enhanced along with the increase of the amount of sulfuric acid. The isosbestic point was observed at $535 \mathrm{~nm}$, which suggests the presence of the two inter 


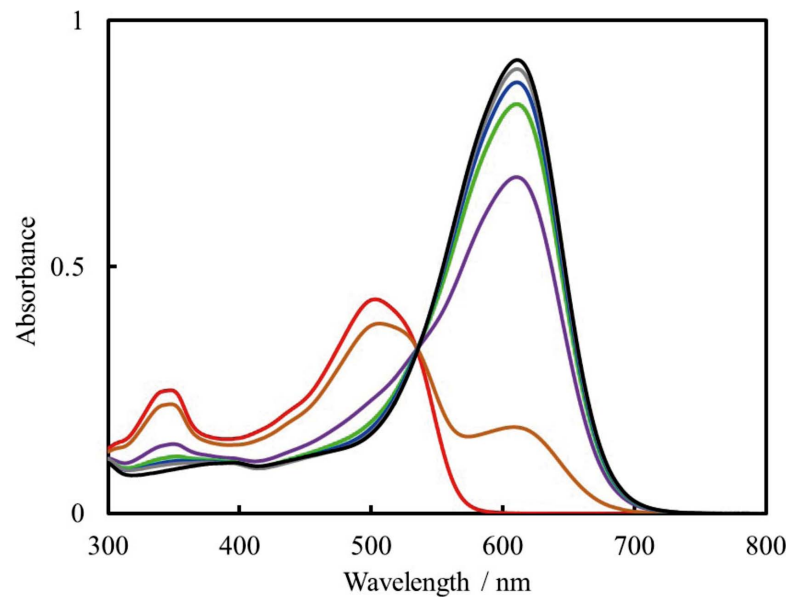

Fig. 2 UV-Vis spectra of acetonitrile solutions of Sudan III with the addition of various volumes of sulfuric acid. Red, $0 \mathrm{~mL}$; orange, $0.004 \mathrm{~mL}$; purple, $0.01 \mathrm{~mL}$, green, $0.02 \mathrm{~mL}$; blue, $0.03 \mathrm{~mL}$; gray, $0.04 \mathrm{~mL}$; black, $0.1 \mathrm{~mL}$ of sulfuric acid were added.

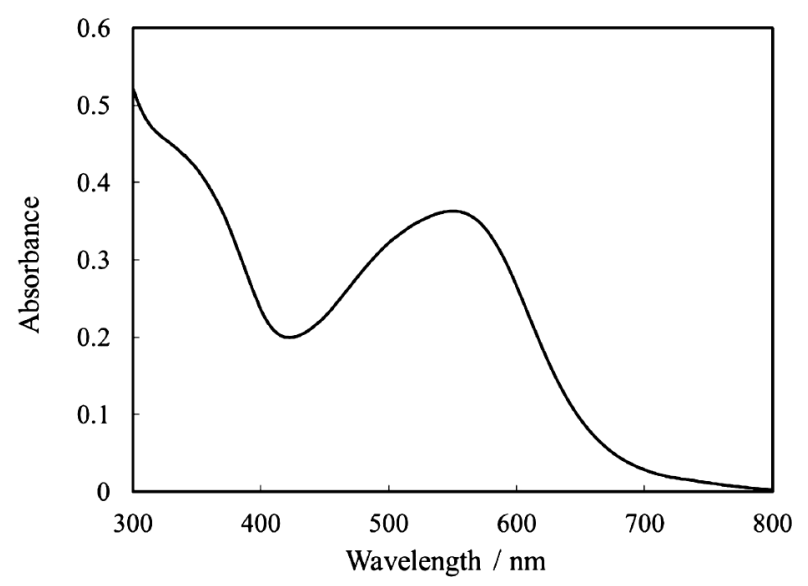

Fig. 3 UV-Vis spectrum of acetonitrile solutions of Sudan III with the addition of an aqueous solution of sodium hydroxide $(2 \mathrm{M})$.

converting species in this system. When a few drops of water were added to the blue solution, the color changed back to orange reversibly.

When the other organic solvents, such as methanol, ethanol, 2-propanol, acetone, dimethyl sulfoxide, were used, in place of acetonitrile, a color change did not occur upon the addition of $0.04 \mathrm{~mL}$ of sulfuric acid. These observations suggest that the hydroxyl, ether, or ketone group including oxygen atom interfered with the interaction between the dye and sulfuric acid. When acids other than sulfuric acid, such as hydrochloric acid, hydrofluoric acid, nitric acid, phosphoric acid, formic acid and acetic acid, were added to the acetonitrile solution of Sudan III, no acid caused the color-change of the solution as sulfuric acid even as high concentration as we can purchase. These facts indicate that the presented system, the acetonitrile solution of Sudan III, is the most suitable for observing the color-change phenomenon.

When the aqueous solution of sodium hydroxide with a concentration of $2 \mathrm{M}$ was added to the acetonitrile solution of Sudan III, the UV-Vis spectrum (shown in Fig. 3) was different from the spectra upon the addition of sulfuric acid, as shown in Fig. 2. This observation leads to the conclusion that the color-

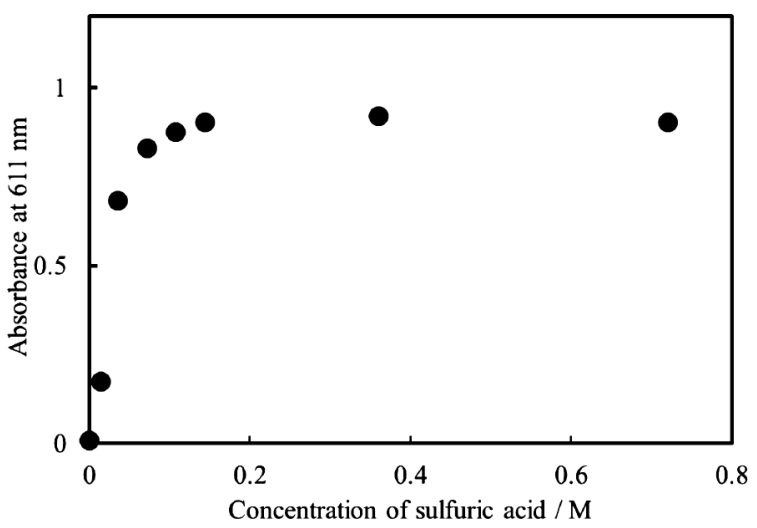

Fig. 4 Relationship between the absorbance at $611 \mathrm{~nm}$ and the sulfuric acid concentration of the solution.

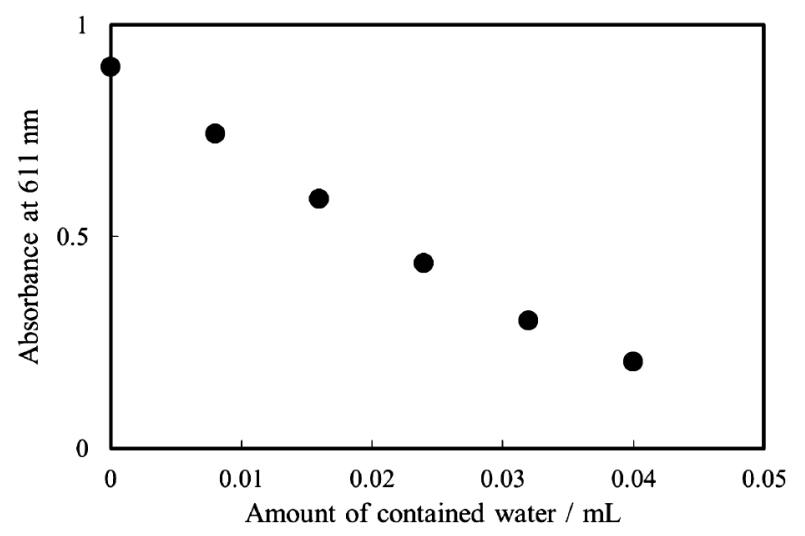

Fig. 5 Relationship between the absorbance at $611 \mathrm{~nm}$ of the dye solution with constant sulfuric acid concentration $(0.144 \mathrm{M})$ and the water content.

change mechanism of Sudan III against sulfuric acid is due to protonation of the dye by sulfuric acid.

\section{Quantification of sulfuric acid}

The relationship between the absorbance at $611 \mathrm{~nm}$ and the sulfuric acid concentration of the solution is shown in Fig. 4. The absorbance reaches a plateau at over $0.36 \mathrm{M}$ of the sulfuric acid concentration. A protonated form of Sudan III has an absorption maximum at $611 \mathrm{~nm}$; on the other hand, an unprotonated form of the dye does not have any absorption at this wavelength. Then, the absorption at $611 \mathrm{~nm}$ reflects the concentration of the protonated form of the dye directly. An HPLC analysis indicated that the reagent includes about $2.2 \%$ of some impurity. Then, the molar absorbance constant of the protonated form of the dye at the absorption maximum is estimated to be $\varepsilon=8.29 \times 10^{4} \mathrm{~L} \mathrm{~mol}^{-1} \mathrm{~cm}^{-1}$ by the LambertBeer law, using the initial concentration of the dye $(1.11 \times$ $\left.10^{-5} \mathrm{M}\right)$ and the absorbance value at the plateau region.

Arnett and Douty have shown that in a non-aqueous solvent (sulfolane) system, small quantities of water reduce the acidities of a sulfuric acid solution. ${ }^{17}$ The presented system looks similar to this combination, since acetonitrile is also the aprotic solvent, and this system is a non-aqueous system. Therefore, the influence of the water content was estimated. The absorbance at $611 \mathrm{~nm}$ of the dye solution with a constant sulfuric acid concentration $(0.144 \mathrm{M})$ containing various amounts of water was measured; the relationship between the absorbance and the 


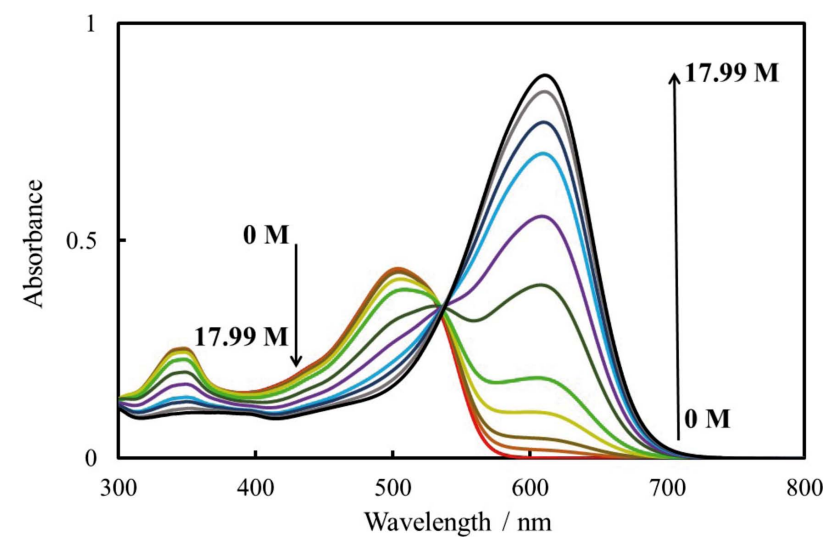

Fig. 6 UV-Vis spectra of acetonitrile solutions of Sudan III with the addition of a constant volume $(0.04 \mathrm{~mL})$ of sulfuric acid sample with various concentrations.

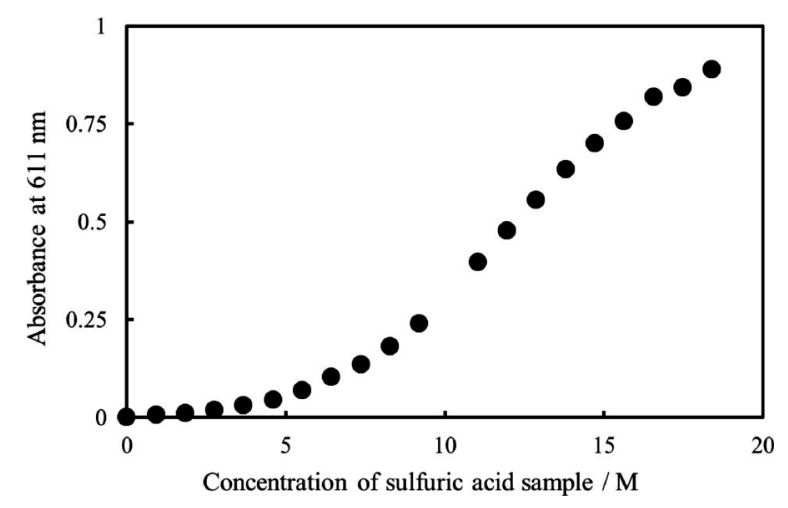

Fig. 7 Relationship between the absorbance at $611 \mathrm{~nm}$ and the concentration of added sulfuric acid samples.

amount of water is shown in Fig. 5. The absorbance at $611 \mathrm{~nm}$, corresponding to the protonated form of the dye, decreased as the water content increased, even though the concentration of the dye and sulfuric acid was kept constant. This phenomenon suggests that water plays the role of a base competing with the dye for protons from the sulfuric acid in this system, which is governed by the same mechanism as that which Arnett and Douty proposed.

From the observation described above, the amount of water that is initially contained in sulfuric acid can also affect the protonation degree of the dye. Figure 6 shows the UV-Vis spectra of acetonitrile solutions of Sudan III with the addition of a constant volume $(0.04 \mathrm{~mL})$ of sulfuric acid with various concentrations (the concentration was adjusted by water). Since the spectrum profiles are the same as those in Fig. 2, the involved species are the same in case the water content increases. Figure 7 shows the relationship between the absorbance at $611 \mathrm{~nm}$ and the concentration of an added sulfuric acid sample. The plots exhibit a sigmoidal relationship, which differ from those in Fig. 4. This difference may be due to the presence of water, which suggests that the small amount of water contained in sulfuric acid sample plays a key role on the protonation degree of the dye by competing with the dye for protons.

The observation described above indicates a possibility that the proposed system has a potential for the quantification of sulfuric acid samples. As shown in Fig. 7, the protonation
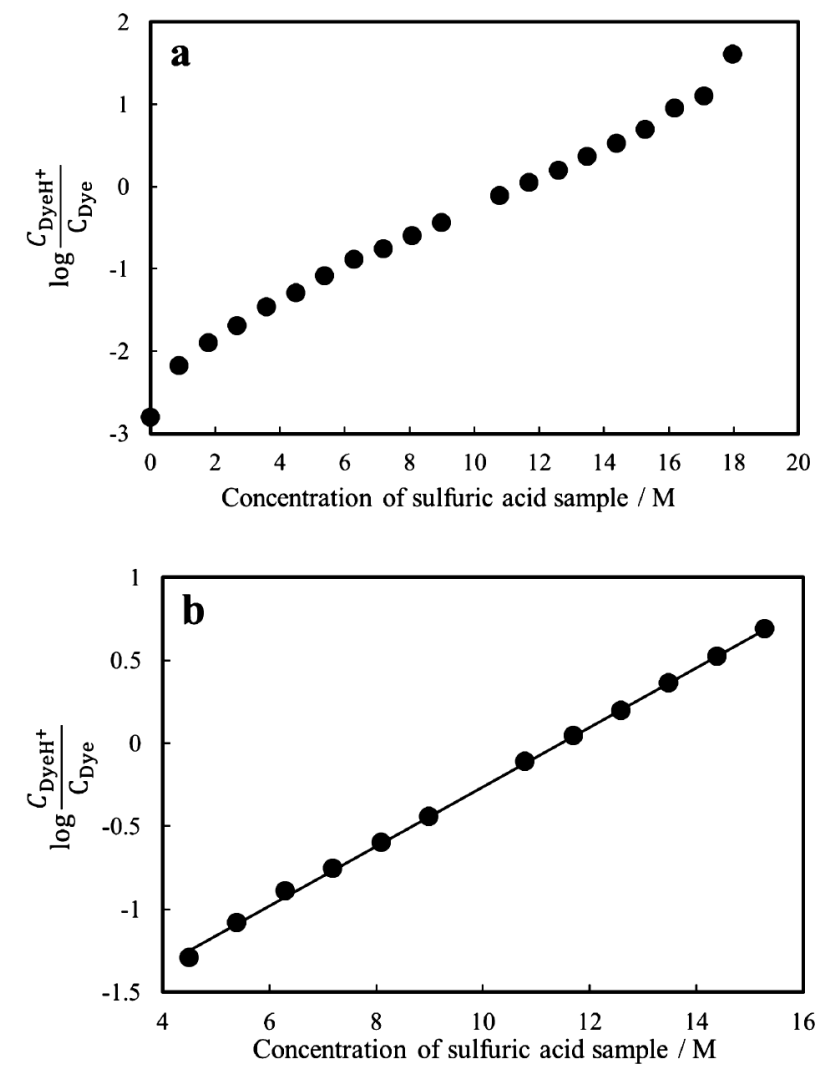

Fig. 8 Relationship between $\log \left(C_{\mathrm{DyeH}^{+}} / C_{\text {Dye }}\right)$ and the concentration of added sulfuric acid samples. (a) Overview, (b) magnified view of within a range of $4.5-15.3 \mathrm{M}$ of sulfuric acid samples.

degree of the dye and the concentration of sulfuric acid samples does not have a liner correlation. To quantify the sulfuric acid concentration, the Hammett acidity function is introduced to the present system. The Hammett acidity function, $H_{0}$, is a parameter of the protonation ability of acid in a non-ideal solution, and is defined as follows ${ }^{18}$ : in the equilibrium as given by the Eq. (1), the dissociate constant, $K_{\mathrm{BH}^{+}}$, of the conjugate acid $\mathrm{BH}^{+}$of base $\mathrm{B}$ is given by Eq. (2) in terms of activity $a$ :

$$
\begin{aligned}
& \mathrm{B}+\mathrm{H}^{+} \rightleftharpoons \mathrm{BH}^{+} \\
& K_{\mathrm{BH}^{+}}=\frac{a_{\mathrm{B}} a_{\mathrm{H}^{+}}}{a_{\mathrm{BH}^{+}}}
\end{aligned}
$$

Taking $\operatorname{logs}$ and substituting $a$ for the concentration $C$ and the molar activity coefficient, $f$, Eq. (2) gives Eq. (3), and $H_{0}$ is defined by Eq. (4):

$$
\begin{aligned}
& \mathrm{p} K_{\mathrm{BH}^{+}}=\log \frac{C_{\mathrm{BH}^{+}}}{C_{\mathrm{B}}}-\log a_{\mathrm{H}^{+}} \frac{f_{\mathrm{B}}}{f_{\mathrm{BH}^{+}}} \\
& H_{0}=-\log a_{\mathrm{H}^{+}} \frac{f_{\mathrm{B}}}{f_{\mathrm{BH}^{+}}}
\end{aligned}
$$

In the present system, Sudan III corresponds to the base, B and is signified as "Dye". Equation (3) can be converted into Eq. (5) using $H_{0}$ :

$$
\log \frac{C_{\mathrm{DyeH}^{+}}}{C_{\text {Dye }}}=-H_{0}+\mathrm{p} K_{\text {DyeH }^{+}}
$$


Table 1 Comparison of the quantification results obtained from the proposed method and titration

\begin{tabular}{ccc}
\hline Sample & Proposed method $^{\mathrm{a}}$ & Titration \\
\hline A & $17.19 \pm 0.86$ & 16.98 \\
B & $15.35 \pm 0.69$ & 15.37 \\
C & $11.14 \pm 1.61$ & 11.27 \\
\hline
\end{tabular}

a. $n=3$, mean $\pm \operatorname{RSD}(\%)$.

Herein, $C_{\mathrm{DyeH}^{+}}$and $C_{\text {Dye }}$ represent the concentrations of the protonated form of the dye and the unprotonated form of the dye, respectively. The ionization ratio, $C_{\mathrm{DyeH}^{+}} / C_{\mathrm{Dye}}$, is experimentally available from UV-Vis spectroscopic measurements while monitoring the absorbance at $611 \mathrm{~nm}$, signified as $A_{611}$. Since the unprotonated form of the dye has no absorption, $A_{611}$ is in proportion to $C_{\mathrm{DyeH}^{+}}$, and $C_{\mathrm{DyeH}^{+}} / C_{\text {Dye }}$ can be described in Eq. (6) using $A_{611}$ and the plateau value, $A_{\infty}$, observed in Fig. 4:

$$
\frac{C_{\mathrm{DyeH}^{+}}}{C_{\text {Dye }}}=\frac{A_{611}}{A_{\infty}-A_{611}}
$$

Since $\mathrm{p} K_{\mathrm{DyeH}^{+}}$is constant, the ionization ratio varies as a function of $H_{0}$. If the $H_{0}$ value depends on the concentration of added sulfuric acid, some correlation between $\log \left(C_{\mathrm{DyeH}^{+}} / C_{\text {Dye }}\right)$ and the concentration of added sulfuric acid samples should be obtained. As shown in Fig. 8a, relationship between $\log \left(C_{\mathrm{DyeH}^{+}} / C_{\text {Dye }}\right)$ and the concentration of added sulfuric acid samples shows a non-liner correlation under $4 \mathrm{M}$ and over $16 \mathrm{M}$. On the other hand, within a range of $4.5-15.3 \mathrm{M}$, a liner correlation was observed, as shown in Fig. 8b. To utilize this liner part for the quantification of a higher concentration sulfuric acid sample, the sample concentration was apparently decreased by the addition of a little volume of water to the dye solution initially. When the sulfuric acid sample was added to the acetonitrile solution of Sudan III containing 0.8 vol\% of water, the plot of $\log \left(C_{\mathrm{DyeH}^{+}} /\right.$ $\left.C_{\text {Dye }}\right) v s$. the concentration of added sulfuric acid sample shows a liner correlation within a range of 6.5-17.99 M (17.99 M of sample is not diluted sample). The liner calibration curve, $y=1.713 x-2.2065$, was obtained in this concentration range; it showed an excellent liner correlation $\left(R^{2}=0.999\right)$. In this way, the initial addition of water to the dye solution enabled us to quantify the concentration of the concentrated sulfuric acid. The proposed method was applied to the quantification of three sulfuric acid samples with unknown concentrations. Samples were also analyzed by the titration method. As shown in Table 1, the analytical results obtained by the proposed method almost agreed with the titration results.

\section{Conclusions}

In this report, the color-change phenomenon of Sudan III against sulfuric acid is described concerning an acetonitrile solution. It is shown that the color-change phenomenon is due to protonation, and that the system is applicable to the quantification of sulfuric acid by introducing the Hammett acidity function. The advantages of the proposed quantification method are that it can be used for the rapid and easy quantification of concentrated sulfuric acid. It requires quite small sample amount, $0.04 \mathrm{~mL}$. This method can be helpful, especially in forensic analyses and industrial quality checks.

\section{References}

1. P. Batamack and J. Fraissard, Catal. Lett., 1997, 46, 129.

2. M. Canakci and J. V. Gerpen, Trans. AESE, 1999, 42, 1203.

3. E. Lotero, Y. Liu, D. E. Lopez, K. Suwannakarn, D. A. Bruce, and J. G. Goodwin Jr., Ind. Eng. Chem. Res., 2005, 44, 5353.

4. M. Zhou, V. Frydman, and L. Frydman, J. Phys. Chem., 1996, 100, 19280.

5. E. W. Matshes, K. A. Taylor, and V. J. Rao, Am. J. Forensic Med. Pathol., 2008, 29, 340.

6. M. Burge, J. C. Hunsaker III, and G. J. Davis, Forensic Sci. Med. Pathol., 2009, 5, 298.

7. S. Kurata and M. Nagai, Energy Fuels, 2004, 18, 1220.

8. T. Kaneko, H. Yoshida, and S. Suzuki, Forensic Sci. Int., 2008, 177, 112.

9. Z. Wang, W. Li, and P. Lu, Sens. Actuators, B, 2004, 99, 264.

10. T. Sakurai, S. Kurata, and K. Ogino, Anal. Sci., 2015, $31,51$.

11. H. F. Dos Santos, L. F. C. De Oliveira, S. D. Dantas, P. S. Santos, and W. B. De Almeida, Int. J. Quant. Chem., 2000, 80, 1076.

12. F. Calbiani, M. Careri, L. Elviri, A. Mangia, L. Pistarà, and I. Zagnoni, J. Chromatogr. A, 2004, 1042, 123.

13. M. Zhou, X. Chen, Y. Xu, J. Qu, L. Jiao, H. Zhang, H. Chen, and X. Chen, Dyes Pigm., 2013, 99, 120.

14. C. V. D. Anibal, I. Ruisánchez, and M. P. Callao, Food Chem., 2011, 124, 1239.

15. S. D. Harvey, G. W. Buchko, R. B. Lucke, C. W. Wright, A. M. Melville, A. J. Scott, and B. W. Wright, Dyes Pigm., 2009, 82, 307.

16. C. V. Di Anibal, M. P. Callao, and I. Ruisánchez, Talanta, 2011, 86, 316.

17. E. M. Arnett and C. F. Douty, J. Am. Chem. Soc., 1964, 86, 409.

18. R. A. Cox and K. Yates, Can. J. Chem., 1983, 61, 2225. 